\title{
Upregulation of the EMT marker vimentin is associated with poor clinical outcome in acute myeloid leukemia
}

\author{
Sharon Wu${ }^{\dagger}$, Yang Dư ${ }^{\dagger}$, John Beckford and Houda Alachkar ${ }^{*}$
}

\begin{abstract}
Background: Vimentin (VIM) is a type III intermediate filament that maintains cell integrity, and is involved in cell migration, motility and adhesion. When overexpressed in solid cancers, vimentin drives epithelial to mesenchymal transition (EMT) and ultimately, metastasis. The effects of its overexpression in AML are unclear.

Methods: In this study, we analyzed the TCGA data of 173 AML patients for which complete clinical and expression data were available. In this analysis, we assessed the association between VIM mRNA expression and patient's clinical and molecular characteristics including clinical outcome.

Results: VIM overexpression was associated with higher white blood count $(<p=0.0001)$. Patients with high VIM expression have worse overall survival (OS) and disease-free survival (DFS) compared with patients with low VIM expression (median OS; 7.95 months vs 19.2 months; $p=0.029$ ). After age-stratification, high VIM expression was significantly associated with worse overall survival in older patients (age $\geq 60$; median OS: 5.4 vs 9.9 months: $p=0.0257$ ) but not in younger patients (age < 60). In stratification analysis according to cytogenetic status, high VIM expression was significantly associated with shorter OS (7.95 vs 24.6 months: $p=0.0102)$ in cytogenetically normal, but not in cytogenetic abnormal AML.
\end{abstract}

Conclusions: Collectively, the data indicate that overexpression of the EMT marker vimentin is associated with poor clinical outcome in older patients with cytogenetically normal AML; and therefore may play a role in this disease.

Keywords: Vimentin, AML, Overall survival, EMT

\section{Background}

Acute myeloid leukemia (AML) is a myeloid lineage malignancy characterized by invasion of the bone marrow and blood by poorly differentiated cells with enhanced proliferative capabilities. Deregulation of various kinases, transcription factors, apoptotic regulators, epigenetic modifiers, etc. and their roles in AML progression have been rigorously studied and are well understood [1]. The deregulation of cytoskeletal proteins particularly those involved in the epithelial to mesenchymal transition (EMT) is well investigated

\footnotetext{
*Correspondence: alachkar@usc.edu

tSharon Wu and Yang Du contributed equally to this work

School of Pharmacy, University of Southern California, Los Angeles, CA, USA
}

in solid cancers but not in hematological malignancies such as AML. Vimentin (encoded by VIM) a type III intermediate filament, forms the cytoskeletal network with microtubules and microfilaments. It is the predominant intermediate filament in mesenchymal cells and leukocytes [2,3]. A highly conserved protein, vimentin participates in crucial cellular processes such as cell motility, migration and adhesion as well as promotes resistance to various cell stressors $[2,4,5]$. Furthermore, though vimentin is a cytoskeletal protein, it has been shown to be involved in apoptotic progression [4, 6-8]. During apoptosis, vimentin is cleaved by caspase- $3,-7$ and -6 resulting in cytoskeletal collapse and is thought to be the basis of the morphological changes that occur in a cell undergoing apoptosis [7]. Direct phosphorylation of vimentin by $A K T 1$, a known cancer 
promoting kinase, protects it from caspase mediated cleavage and blocks apoptotic progression $[9,10]$. Given that vimentin regulates cell migration and adhesion, which are crucial processes in cancer progression, it has long been seen as a factor in cancer progression. Vimentin overexpression has been reported in cancers of the prostate, gastrointestinal tract, breast and many others [10-12]. In solid cancers, vimentin has been shown to promote metastatic progression by participating in the cytoskeletal reorganization that occurs during EMT as well as regulate pro-EMT signaling pathways [10]. Vimentin has also been used as a marker for pre-metastatic cells undergoing EMT and therefore, high vimentin expression is associated with worse outcomes in patients with solid cancer [11].

Vimentin is recognized as a player in solid cancer progression but its function in blood cancers, particularly AML, is less clear as leukemic cells do not undergo EMT. We speculate that in AML, vimentin may act as a negative regulator of apoptosis, promote cell motility, confer increased resistance to various stressors as well as regulate signaling networks that promote leukemic cell survival. Based on this speculation, we hypothesize that high VIM expression is associated with more aggressive disease and worse outcomes in AML patients.

\section{Methods}

\section{Patients and treatment}

Data on patients with complete clinical and RNA expression data from the cancer genome atlas (TCGA) dataset were included in this study. We identified 173 patients with AML (median age 58 years; range 18-88). These patients were diagnosed and received treatment according to National Comprehensive Cancer Network (NCCN) guidelines between November 2001 and March 2010 [13]. Patients in the intermediate and poor cytogenetic risk groups did not receive uniform treatment: they were included in clinical trials and received allogeneic stem cell transplants whenever applicable or when matched donors were available. In our analysis, we adjusted for transplant status [13]. In total, 91 patients $(52.6 \%)$ were aged $<60$ years and 82 patients $(47.4 \%)$ were aged 60 $\geq$ years. The diagnosis of AML as well as risk group stratification were done according to NCCN guidelines. The patients were assigned subtype classifications according to the French-American-British (FAB) classifications. The patients included in the study were assessed for gene expression as well as somatic mutations frequently found in AML, such as FLT3, NPM1, IDH1/2, TET, etc. Patient clinical, gene expression ( $\mathrm{Z}$ score), mutations, gene methylation and survival data were downloaded from the TCGA database on March 10, 2018 via cBioportal $[14,15]$.

\section{Gene expression analyses}

Publicly available analyzed RNA sequencing data were downloaded from TCGA. Expression values (Z-scores) were used to dichotomize patients into two groups based on VIM expression data $\mathrm{Z} \geq 1$ and $\mathrm{Z}<1$. In additional analysis we divided patients into $\mathrm{Z} \geq 2$ and $\mathrm{Z}<2$.

\section{Statistical analyses}

The time between diagnosis and removal from study due to lack of complete remission, relapse, or death was defined as disease free survival (DFS). The time between diagnosis and death due to any reason was defined as overall survival (OS). Kaplan-Meier survival were generated for the comparison of overall and disease-free survival between patients with $\mathrm{Z} \geq 1$ and $\mathrm{Z}<1 \quad V I M$ expression. In order to determine associations between VIM expression levels and patient clinical/molecular characteristics, Mann-Whitney U's non-parametric and Fisher's exact test were used for continuous and categorical variables, respectively, using STATA 12.0 SE. Figures were generated using GraphPad Prism software package (ver. 5.0; GraphPad Software Inc., La Jolla, CA, USA). The Stata 12.0 SE software was utilized to perform multivariate analysis using the Cox Proportional Hazards Model to assess the association between VIM expression and OS as well as DFS after adjusting for other clinical factors. We also conducted survival analysis by dichotomizing patients according to age $(<60)$ into younger and older $(\geq 60)$ and excluding patients with t15:17 inversion. A statistical cut-off of $\mathrm{p}<0.05$ was used for inclusion of variables from univariate analysis to multivariate analysis.

\section{Results \\ VIM expression in AML samples}

VIM mRNA expression (RNA Seq V2 RSEM) data were downloaded from TCGA; and $\log 2$ transformed. Histograms depicting the distribution of these mRNA $\log 2$-transformed data and VIM Z scores are shown in Fig. 1a, b, as well as a scatter plot of VIM log2-transformed mRNA expression against VIM Z-score (Fig. 1c: adjusted- $R^{2}: 0.775, p \leq 0.001$ ). Based on the distribution of VIM Z scores, we used a $\mathrm{Z}$ score cut off of 1 and 2 for further analysis.

In order to assess VIM expression in primitive progenitor cells, we analyzed the AML-GSE30377 data. This set provides gene expression data of cells obtained from 23 patients with AML and sorted into stem cells and progenitors according to CD34 and CD38 markers. VIM expression was not found to be higher in the sorted population enriched for the stem cells and the progenitors $(\mathrm{CD} 34+/ \mathrm{CD} 38-)$ and $(\mathrm{CD} 34+/ \mathrm{CD} 38+)$ populations. 

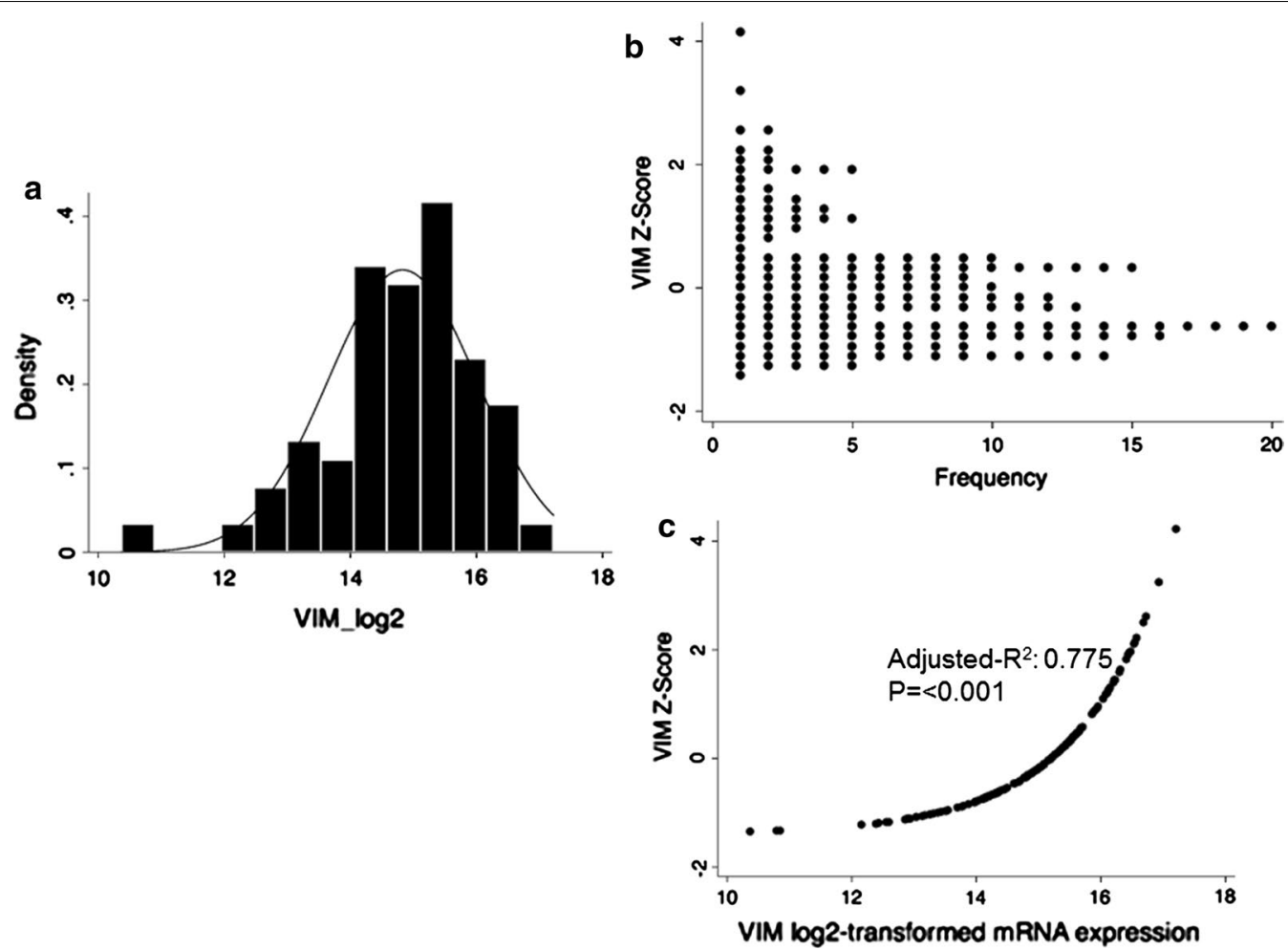

Fig. 1 VIM Z-score and mRNA expression distribution. a Histogram of VIM (log2 transformed) mRNA expression; b dotplot of VIM mRNA expression Z-score; c scatterplot of VIM log2 mRNA expression vs VIM Z-scores

However, we found that VIM was significantly lower in the CD34-/CD38- cells compared to unsorted cells (Additional file 1: Figure S1).

\section{Association between VIM expression and patient primary characteristics}

We analyzed VIM mRNA expression (log2-transformed) of AML patients based on cytogenetic risk and cytogenetic status. We found that median VIM mRNA expression was significantly higher in cytogenetically normal (CN-AML) patients compared to cytogenetically abnormal (CA-AML) patients (Fig. 2a: 15.4 vs 14.8; $\mathrm{p}=0.0007$ ). Additionally, median VIM mRNA expression was significantly lower in patients with poor cytogenetic risk compared to intermediate and favorable risk (Fig. 2b: $\mathrm{p}=0.0011$ ). Median VIM mRNA expression was significantly lower in M0 and M2 FAB classes compared to M3, M4 and M5 FAB classes of AML (Fig. 2c: $\mathrm{p} \leq 0.0001$ ).

Next we categorized patients according to VIM Z score into high $(Z \geq 1)$ and low $(Z<1)$ and assessed the association between high VIM expression $(Z \geq 1)$ and patients clinical characteristics (Table 1). We found that 28 patients $(16 \%)$ have high VIM expression $(\mathrm{Z} \geq 1)$. Patients with high VIM have higher WBC counts (median: 69.2 vs 12.6, $\mathrm{p}<0.001$ ) and higher bone marrow blasts (median
\%: 82.5 vs $71, \mathrm{p}=0.017)$ than patients with low VIM. High VIM expression was also significantly associated with age (median: 63.5 vs $57 ; \mathrm{p}=0.048$ ), normal cytogenetic (\%: 71.4 vs $41.4, \mathrm{p}=0.003)$ and transplant status (\%: 21.4 vs $46.2 ; \mathrm{p}=0.021)$. There was no significant association between high VIM expression $(\mathrm{Z} \geq 1)$ and FAB subtype, sex, or median peripheral blood blast percentage. However, when we considered patient with $\mathrm{Z} \geq 2$ for high VIM expression, we found that high VIM is associated with advanced age (median: 69 vs $57, \mathrm{p}=0.036$ ), median white blood cell count (median: 69.2 vs $15.1 ; \mathrm{p}=0.016$ ) and increased peripheral blast counts (median \%: 65 vs 33, $\mathrm{p}=0.022$ ) (Additional file 1: Table S1).

\section{Association between VIM expression and patient mutational status}

Furthermore, we analyzed VIM expression according to patient's mutational status (Table 2). VIM high expression $(\mathrm{Z} \geq 1)$ was found to be associated with NPM1 mutation (\%: $46.4 \%$ vs $24.1 \%$; p value: 0.021 ), but this association was lost when considering Z-score $\geq 2$ (\%: 37.5 vs $27.3 \%$; p value: 0.687 , Additional file 1: Table S2). No other association was found between VIM expression and patient's mutational status. 

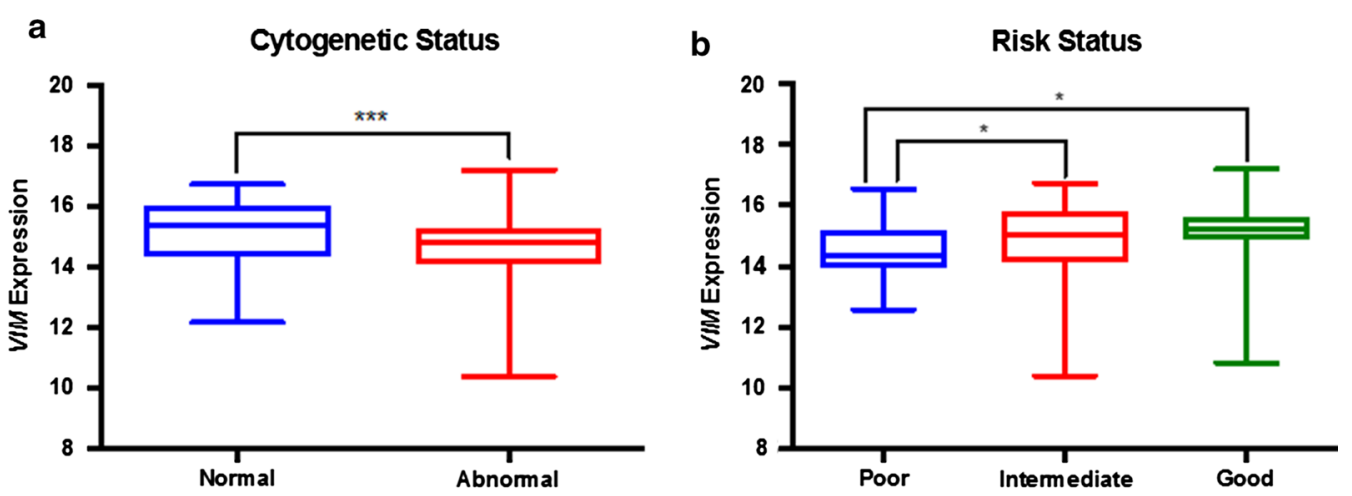

C VIM Expression in FAB subtypes

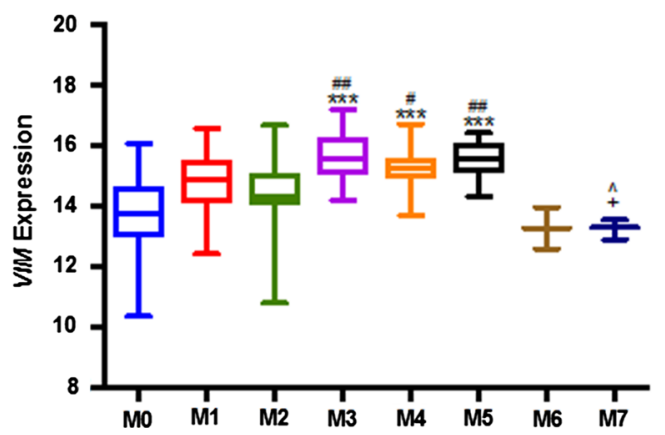

Fig. 2 VIM mRNA expression in patients with AML according to cytogenetic, risk and FAB classifications. VIM log2 mRNA expression categorized by a cytogenetic status, $\mathbf{b}$ risk status and $\mathbf{c}$ FAB classification: asterisk compared to M0, hash symbol compared to M2, plus sign compared to M3 and cap symbol compared to M5. ${ }^{*} p<0.05 ;{ }^{* *} p<0.001$

\section{Patients with high VIM expression have shorter overall and disease-free survival}

We compared overall survival and disease-free survival (OS and DFS, respectively) between patients with high $(Z \geq 1)$ and low $(Z<1)$ VIM expression. We found that patients with high VIM expression $(Z \geq 1)$ had significantly shorter median OS than patients with low VIM expression (median: 7.95 months vs 19.2 months; $\mathrm{p}=0.029$ ) (Fig. 3a). Similarly, high VIM expression patients had significantly shorter DFS compared with low VIM expression patients (median: 5.65 months vs 10.8 months; $\mathrm{p}=0.0138$ ) (Fig. $3 \mathrm{~b}$ ). After age stratification, high VIM expression was still significantly associated with worse overall survival in older patients (Fig. 4a: age $\geq 60$-median 5.4 vs 9.9 months: $\mathrm{p}=0.0257$ ) but not in younger patients (Fig. 4b: age $<60$ ). Importantly, when we stratified patients according to the cytogenetic status; we found that in CN-AML, but not in CAAML, high VIM expression $(\mathrm{Z} \geq 1)$ was significantly associated with shorter overall survival (Fig. 5a, b: 7.95 vs 24.6 months: $\mathrm{p}=0.0102)$. Stratification of patients according to transplant status showed a similar trend of an association between high VIM expression and shorter OS, however it did not reach statistical significance. (no transplant-Additional file 1: Figure S2A: 6.9 vs 10.2 months: $\mathrm{p}=0.1607$; received transplant-Additional file 1: Figure S2B: 24.8 vs 30.6 months: $\mathrm{p}=0.5265$ ).

We analyzed the association between VIM expression and clinical outcomes in three other datasets: two Metzeler and one Bullinger datasets. Because $\mathrm{Z}$ scores were not available in these datasets, we dichotomized patients according to median VIM expression into high (above median) and low (below median). High VIM expression was significantly associated with worse overall survival (Fig. 6a: $n=163$ : 263 vs 657 days; p value: 0.0396). Similarly, the second Metzeler dataset (Fig. $6 \mathrm{~b}: \mathrm{n}=79$ : 392 vs 624 days; $p$ value: 0.1002 ) showed a non-significant but similar trend towards worse overall survival associated with high VIM expression, both Metzeler datasets include CN-AML patients only [16]. Contrastingly, the Bullinger $(\mathrm{n}=119)$ dataset which includes only 45 patients with CN-AML, showed no significant association between VIM expression and clinical outcome (Fig. 6c) [17].

After adjusting for other risk factors associated with disease free survival, multivariate analyses indicated that high $(\mathrm{Z} \geq 1)$ VIM expression was associated with shorter DFS in patients with AML (HR: 2.42 95\% CI 1.26-4.66; $\mathrm{p}=0.008$, Table 3 ). The association between 
Table 1 Clinical characteristics of 173 AML patients according to VIM expression Z-score $\geq 1$

\begin{tabular}{|c|c|c|c|}
\hline Characteristic & Z-score $(<1)(n=145)$ & Z-score $(\geq 1)(n=28)$ & $\mathrm{p}$ value \\
\hline Age, median (years) & 57 & 63.5 & 0.048 \\
\hline Young ( $<60$ years) & $81(55.9 \%)$ & $10(64.3 \%)$ & 0.063 \\
\hline Old (> 60 years) & $64(44.1 \%)$ & $18(35.7 \%)$ & \\
\hline \multicolumn{4}{|l|}{ Sex } \\
\hline Female (n, \%) & $68(46.9 \%)$ & $13(46.4 \%)$ & $>0.999$ \\
\hline Male (n, \%) & $77(53.1 \%)$ & $15(53.6 \%)$ & \\
\hline \multicolumn{4}{|l|}{$F A B$} \\
\hline $\mathrm{MO}(\mathrm{n}, \%)$ & $15(10.3 \%)$ & $1(3.57 \%)$ & 0.476 \\
\hline$M 1(n, \%)$ & $39(26.9 \%)$ & $5(17.9 \%)$ & 0.353 \\
\hline$M 2(n, \%)$ & $34(23.5 \%)$ & $4(14.3 \%)$ & 0.329 \\
\hline M3 $(n, \%)$ & $11(7.59 \%)$ & $5(17.9 \%)$ & 0.146 \\
\hline M4 $(n, \%)$ & $27(18.6 \%)$ & $7(25.00 \%)$ & 0.446 \\
\hline M5 $(n, \%)$ & $12(8.28 \%)$ & $6(21.4 \%)$ & 0.083 \\
\hline M6 $(n, \%)$ & $2(1.38 \%)$ & $0(0.00 \%)$ & $>0.999$ \\
\hline$M 7(n, \%)$ & $3(2.07 \%)$ & $0(0.00 \%)$ & $>0.999$ \\
\hline WB count, median & 12.6 & 69.2 & $<0.001$ \\
\hline In (WB count), mean & 2.446 & 3.749 & $<0.001$ \\
\hline$\%$ BM blast, median & 71 & 82.5 & 0.017 \\
\hline$\%$ PB blast, median & 34 & 44.5 & 0.253 \\
\hline \multicolumn{4}{|l|}{ Risk status } \\
\hline Poor (n, \%) & $42(29.0 \%)$ & $3(10.7 \%)$ & 0.057 \\
\hline Intermediate (n, \%) & $73(50.3 \%)$ & $19(67.9 \%)$ & 0.091 \\
\hline Good (n, \%) & $28(19.3 \%)$ & $5(17.9 \%)$ & $>0.999$ \\
\hline \multicolumn{4}{|l|}{ Cytogenetic status } \\
\hline Normal $(\mathrm{n}, \%)$ & $60(41.4 \%)$ & $20(71.4 \%)$ & 0.003 \\
\hline Abnormal $(n, \%)$ & $83(57.2 \%)$ & $7(25.0 \%)$ & \\
\hline \multicolumn{4}{|l|}{ Transplant status } \\
\hline No $(n, \%)$ & $78(53.8 \%)$ & $22(78.6 \%)$ & 0.021 \\
\hline Yes (n, \%) & $67(46.2 \%)$ & $6(21.4 \%)$ & \\
\hline
\end{tabular}

Table 2 Expression of VIM (Z-score $\geq 1)$ according to the top mutations present in $A M L(N=173$ patients)

\begin{tabular}{llcr}
\hline Genes & $\begin{array}{l}\text { Z-score }(<\mathbf{1}) \\
(\mathbf{n}=\mathbf{1 4 5})\end{array}$ & $\begin{array}{l}\text { Z-score }(\geq \mathbf{1}) \\
(\mathbf{n}=\mathbf{2 8})\end{array}$ & p value \\
\hline FLT3 (n, \%) & $41(28.3 \%)$ & $8(28.6 \%)$ & $>0.999$ \\
TP53 (n, \%) & $14(9.66 \%)$ & $0(0.00 \%)$ & 0.130 \\
NPM1 (n, \%) & $35(24.1 \%)$ & $13(46.4 \%)$ & 0.021 \\
NRAS (n, \%) & $10(6.90 \%)$ & $2(7.14 \%)$ & $>0.999$ \\
TET2 (n, \%) & $14(9.66 \%)$ & $1(3.57 \%)$ & 0.470 \\
RUNX1 (n, \%) & $14(9.66 \%)$ & $1(3.57 \%)$ & 0.470 \\
CEBPA (n, \%) & $12(8.28 \%)$ & $1(3.57 \%)$ & 0.696 \\
WT1 (n, \%) & $10(6.90 \%)$ & $0(0.00 \%)$ & 0.369 \\
DNMT3A (n, \%) & $34(23.4 \%)$ & $8(28.6 \%)$ & 0.631 \\
IDH1 (n, \%) & $13(8.97 \%)$ & $3(10.7 \%)$ & 0.726 \\
IDH2 (n, \%) & $13(8.97 \%)$ & $4(14.3 \%)$ & 0.484 \\
\hline
\end{tabular}

high $(\mathrm{Z} \geq 1)$ VIM expression and $\mathrm{OS}$ did not reach statistical significance (HR: 1.42 95\% CI 0.85-2.38; $\mathrm{p}=0.178$, Additional file 1: Table S3) in multivariable analysis. However, multivariate analyses showed a significant association between higher VIM expression $(\mathrm{Z} \geq 2)$ and shorter overall survival (HR: 3.99 95\% CI 1.65-9.66; $\mathrm{p}=0.002$ ) (Additional file 1: Table S4).

After age-stratification, high VIM expression $(\mathrm{Z} \geq 1)$ was significantly associated with worse overall survival even after adjustment for other risk factors in older patients (HR: 1.99 95\% CI 1.04-3.81; $\mathrm{p}=0.038$, Table 4) but not younger patients (Additional file 1: Table S5). Similarly, higher VIM expression $(\mathrm{Z} \geq 2)$ was significantly associated with worse overall survival in older patients (HR: 4.27 95\% CI 1.62-11.3; $\mathrm{p}=0.003$, Additional file 1: Table S6). Additionally, when we 

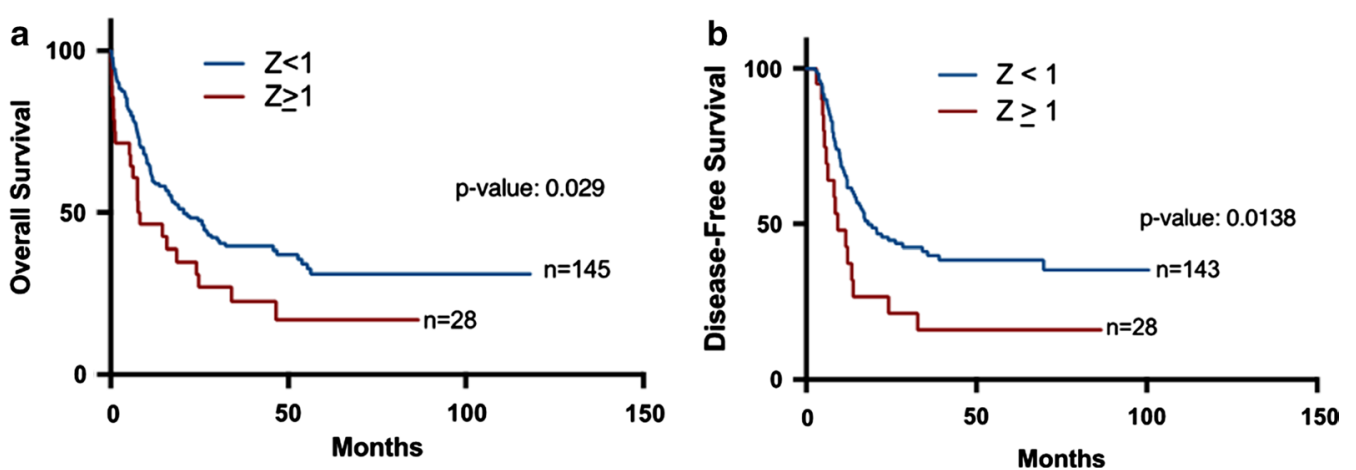

Fig. 3 Survival analysis of AML patients associated with VIM expression. a Overall survival of 173 AML patients with VIM Z-score $\geq 1$ and VIM Z-score $<1$; $\mathbf{b}$ disease-free survival of 171 AML patients with VIM Z-score $\geq 1$ and VIM Z-score $<1$
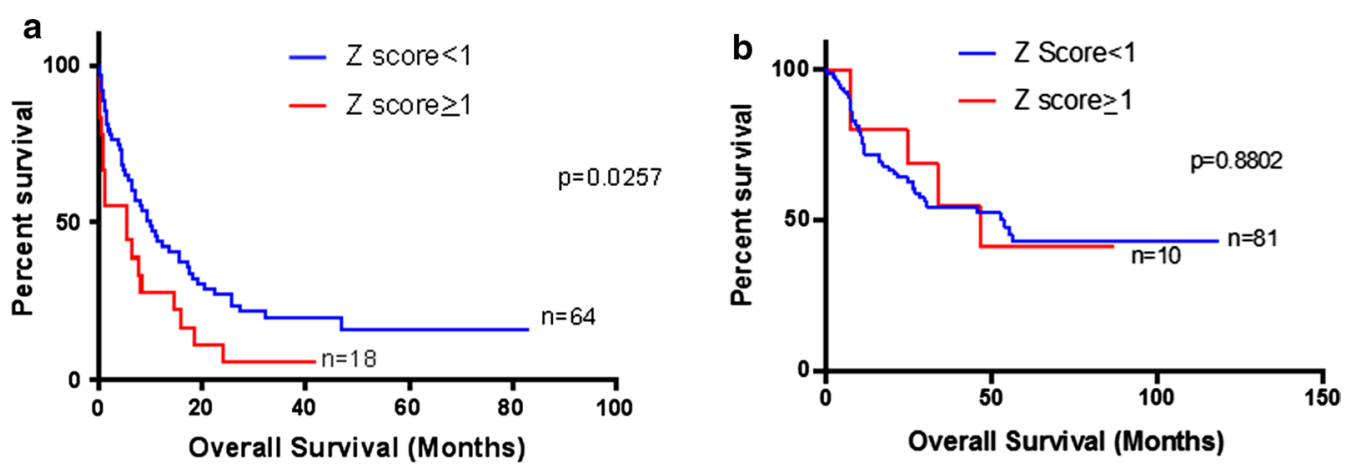

Fig. 4 Survival analysis of AML patients associated with VIM expression after age-stratification. a Overall survival of AML patients with VIM $Z$-score $\geq 1$ and VIM Z-score $<1$ in older patients (age $\geq 60$ ). $\mathbf{b}$ Overall survival of AML patients with VIM Z-score $\geq 1$ and VIM Z-score $<1$ in younger patients (age <60)
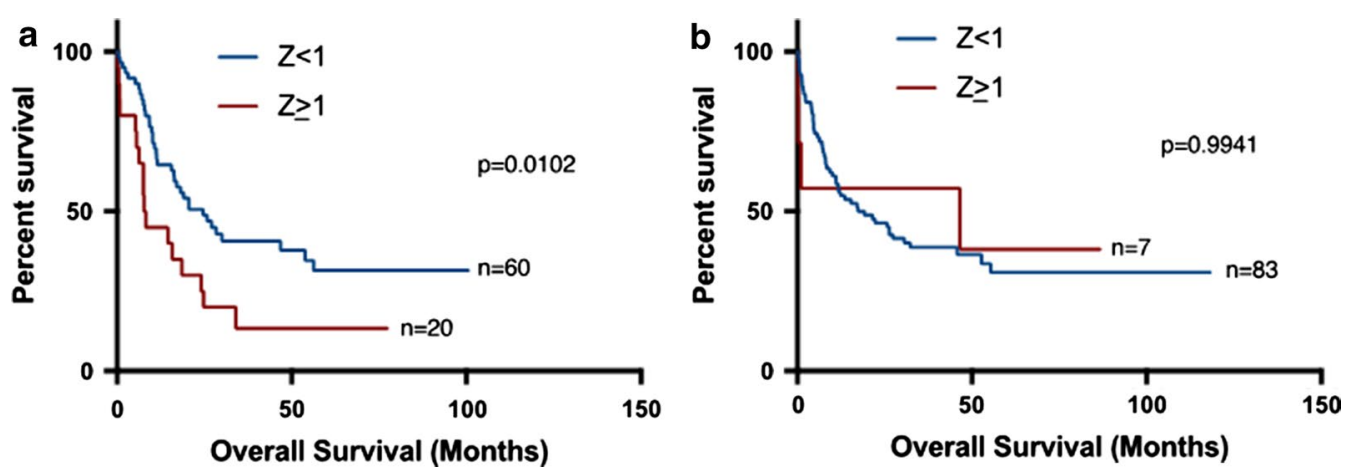

Fig. 5 Survival analysis of AML patients associated with VIM expression after stratification according to cytogenetic status. a Overall survival of AML patients with VIM expression VIM Z-score $\geq 1$ and VIM Z-score $<1$ in cytogenetically normal patients. $\mathbf{b}$ Overall survival of AML patients with VIM expression VIM Z-score $\geq 1$ and VIM Z-score $<1$ in cytogenetically abnormal patients

exclude patients with t15:17 inversions, higher VIM expression $(Z \geq 2)$ remained significantly associated with worse overall survival (HR: $4.3095 \%$ CI $1.60-$ 11.6; $\mathrm{p}=0.004)$.
VIM is hypomethylated in patients with high VIM expression

Because VIM upregulation was not consistently associated with any particular mutation found in AML, we 

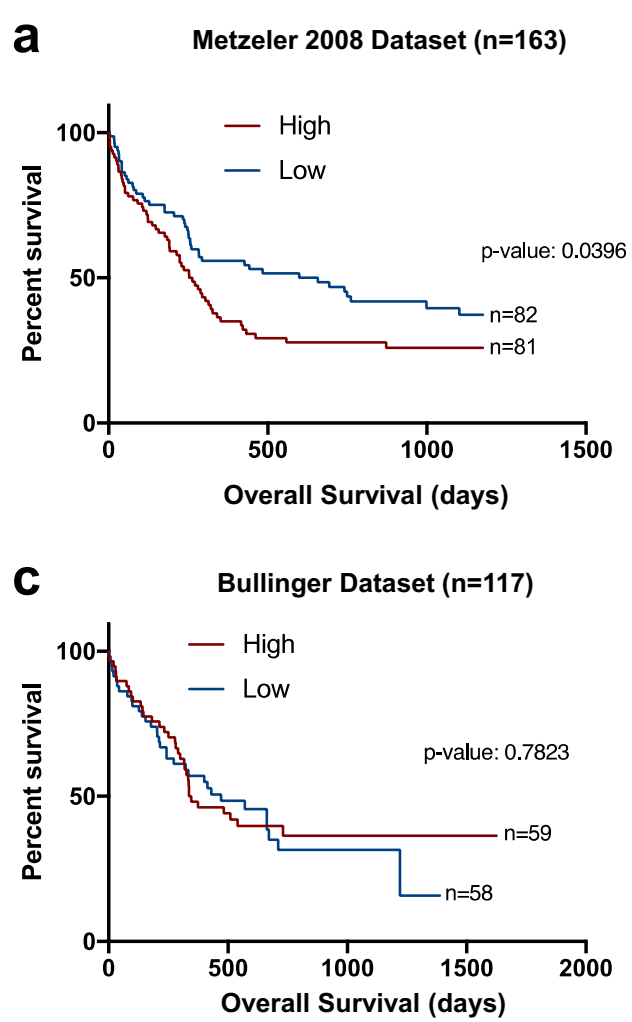

b Metzeler 2008 Dataset $(n=79)$

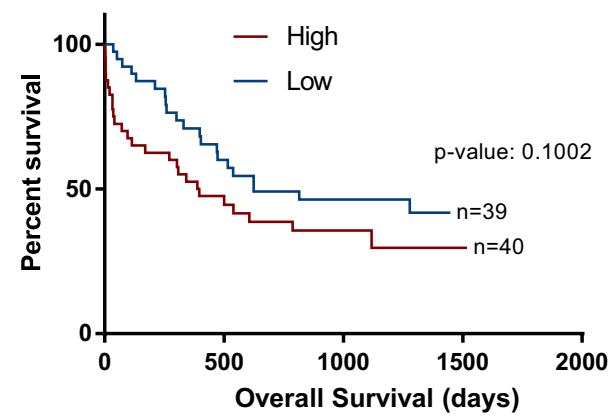

Fig. 6 Survival analysis of patients with AML associated with VIM expression in three different datasets. Overall survival of patients with AML in various datasets after dichotomization of VIM mRNA expression into high and low according to the log-2 median-centered expression: $\mathbf{a}, \mathbf{b}$ CN-AML (Metzeler, Blood, 2008) and c CN-AML and CA-AML (Bullinger, NEJM, 2004)

Table 3 Multivariate analysis of disease-free survival of patients with AML for VIM expression Z-score $\geq 1(N=166)$

\begin{tabular}{lllll}
\hline Variables & Hazard ratio & $\mathbf{9 5 \%} \mathbf{C l}$ & & p value \\
\hline InWBC & 1.05 & 0.87 & 1.27 & 0.633 \\
PB blast & 1.01 & 1.001 & 1.02 & 0.028 \\
Cytogenetic abnormality & 1.82 & 0.83 & 4.00 & 0.134 \\
Cytogenetic risk & & & & \\
$\quad$ Intermediate & 4.76 & 1.73 & 13.1 & 0.002 \\
Poor & 3.85 & 1.49 & 9.90 & 0.005 \\
Transplant status & 0.98 & 0.58 & 1.65 & 0.926 \\
FLT3 & 2.03 & 1.21 & 3.40 & 0.007 \\
VIM & 2.42 & 1.26 & 4.66 & 0.008 \\
\hline
\end{tabular}

speculated that other mechanisms might be involved in regulating this gene. In order to examine whether epigenetic changes might play a role in regulating VIM expression, we compared methylation $\beta$ values between VIM high $(\mathrm{Z} \geq 1)$ and VIM low $(\mathrm{Z}<1)$. We found that $V I M$ methylation was significantly lower in the high VIM patients compared to that in the low VIM patients (Fig. 7; median methylation $\beta$ value: 0.1 vs $0.2, \mathrm{p}=0.0052$ ).

\section{Discussion}

The epithelial to mesenchymal transition (EMT) is an embryonic developmental process in which epithelial cells transition to cells with a mesenchymal phenotype defined by the loss of E-cadherin and gain of vimentin expression markers [18]. EMT has been shown to play oncogenic roles in cancer progression starting from the initiation of the primary tumor thorough metastasis and resistance to cancer treatment [18]. EMT has also been linked to cancer stem cell survival and maintenance. Importantly, the role of EMT in cancer is not limited to cancer of epithelial origin, but also extend to non-epithelial cancers such as glioma and hematological malignancies [19]. In hematological malignancies, several studies have reported on the role of the EMT transcription factors TWIST1 and TWIST2 in both myeloid and lymphoblastic leukemia [19]. Using an inducible MLLAF9-driven AML mouse model, a recent study demonstrated that the expression of MLL-AF9 in hematopoietic stem cells (HSC) drives an invasive and chemoresistant AML expressing EMT markers (such as VIM among others) [20].

VIM is a cytoskeletal protein which overexpression is known to be associated with aggressive disease and 
Table 4 Multivariate analysis of overall survival of AML patients associated with VIM expression Z-score $\geq 1$ in older patients (age $\geq 60 ; n=80$ )

\begin{tabular}{lllll}
\hline Variables & Hazard ratio & $\mathbf{9 5 \%} \mathbf{C l}$ & & p value \\
\hline Age & 1.02 & 0.97 & 1.06 & 0.451 \\
Cytogenetic risk & & & & \\
$\quad$ Intermediate & 0.90 & 0.35 & 2.32 & 0.828 \\
$\quad$ Poor & 3.60 & 1.18 & 11.0 & 0.024 \\
Transplant status & 0.274 & 0.12 & 0.62 & 0.002 \\
DNMT3A & 1.77 & 0.94 & 3.32 & 0.077 \\
RUNX1 & 1.67 & 0.72 & 3.86 & 0.230 \\
TP53 & 1.31 & 0.57 & 3.01 & 0.517 \\
VIM & 1.99 & 1.04 & 3.81 & 0.038 \\
\hline
\end{tabular}

\section{VIM Methylation}

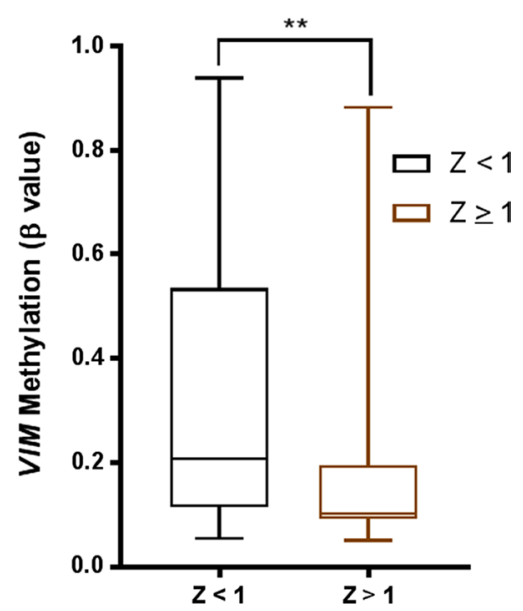

Fig. 7 Associations between methylation and VIM expression. VIM methylation $\beta$ value comparison between patients according to VIM expression ( $Z$-score $\geq 1$ and $<1$ ). A non-parametric MannWhitney $U$ test was used to compare the median of methylation $\beta$ value between the groups ( $p$ value: 0.0052)

worse outcomes in solid cancers such breast, gastrointestinal, and prostate cancers [9-12]. VIM regulates EMT, in part, due to its role in regulating cell migration, cell adhesion, EMT signaling pathways and cytoskeletal reorganization [4]. In this study, we characterized the EMT marker VIM, in the context of AML. As leukemic cells do not undergo EMT, the molecular functions of VIM are less clear in AML. Here we demonstrated that upregulation of VIM is associated with poor overall and disease-free survival in older patients with CN-AML. The clinical outcome of AML in older patients who are unable to receive intensive chemotherapy without unacceptable side effects remains dismal. Less than $15 \%$ of patients older than 60 years of age survive for longer than 5 years; and the median survival of these patients is only
5-10 months [1]. Whether vimentin protein levels correlate with its mRNA levels and similarly with clinical outcome remains to be examined. The lack of association between high VIM and patient's mutational status is particularly interesting, suggesting other mechanisms such as epigenetic modification may be involved in regulating $V I M$ expression. Indeed, we found that patients with high VIM $(\mathrm{Z} \geq 1)$ have significantly lower VIM methylation in comparison with low VIM expression. Consistent with our observation, treatment with 5-Aza-Deoxycytidine, a methylation inhibitor, resulted in several folds increase of vimentin mRNA expression in different colon cancer cell lines [21]. Vimentin expression was also shown to be transactivated by $\beta$-catenin/TCF, binding to a site upstream of vimentin promoter in breast cancer cells [22]. Whether similar mechanisms are present in AML is unknown, however the role of $\beta$-catenin signaling pathway in maintaining leukemia stem cell survival is well established in AML $[23,24]$.

\section{Conclusion}

In conclusion, our results demonstrate that VIM upregulation is associated with poor clinical outcome in AML. The characterization of VIM mRNA expression reveals it as valuable prognostic marker in AML and provides the rationale for further functional and mechanistic investigation into the role of VIM in this disease.

\section{Additional file}

Additional file 1: Figure S1. VIM mRNA expression in sorted AML cells according to their leukemia stem cell markers expression. VIM gene expression data obtained from the GSE30377 dataset, in which leukemia blasts obtained from patients with AML $(n=23)$ were sorted into CD34+CD38-, CD34+CD38+, CD34-CD38-, and CD34-CD38+ populations, VIM mRNA levels were compared between the different sorted cell population and unsorted cells. ${ }^{*} P<0.05$. Figure $\mathbf{S 2}$. Survival analysis of AML patients associated with VIM expression after stratification of transplant status. (A) Overall survival of AML patients with VIM expression VIM Z-score $\geq 1$ and VIM Z-score $<1$ in patients who did not receive transplant. (B) Overall survival of AML patients with VIM expression VIM Z-score $\geq 1$ and VIM Z-score $<1$ in patients who received transplant. Table S1.

Clinical Characteristics of 173 AML Patients According to VIM Expression Z-Score $\geq 2$. Table S2. Expression of VIM (Z-Score $\geq 2$ ) according to the top mutations present in AML ( $N=173$ patients). Table S3. Multivariate Analysis of Overall Survival of AML Patients Associated with VIM Expression Z-Score $\geq 1$ ( $n=169$ ). Table S4. Multivariate Analysis of Overall Survival of AML Patients Associated with VIM Expression Z-Score $>2(n=169)$. Table S5. Multivariate Analysis of Overall Survival of AML Patients Associated with VIM Expression Z-Score $\geq 1$ in young patients (Age $<60 ; n=89$ ). Table S6. Multivariate Analysis of Overall Survival of AML Patients Associated with VIM Expression Z-Score $\geq 2$ in old patients (Age $\geq 60 ; n=80$ ).

\section{Authors' contributions}

SW and YD performed the analysis and generated the figures, SW performed the statistical analysis and contributed to writing the manuscript, JB contributed to data analysis and writing the draft. HA conceived the project, designed the research, supervised the research and written the final draft. All authors read and approved the final manuscript. 


\section{Acknowledgements}

We would like to thank the Bioinformatics core at the Norris medical library, University of Southern California and TCGA. We like to acknowledge the University Of Southern California School Of Pharmacy Seed Fund and USC CTSI funding for Dr. Alachkar.

\section{Competing interests}

The authors declare that they have no competing interests.

\section{Consent for publication}

Not applicable.

\section{Data availability}

The TCGA data analyzed in this study is publicly available from cBioportal.

\section{Ethics approval and consent to participate}

Not applicable, the data are publically de-identified data.

\section{Funding}

This study was funded partialy by CTSI KL-2 funding for Dr. Alachkar.

\section{Publisher's Note}

Springer Nature remains neutral with regard to jurisdictional claims in published maps and institutional affiliations.

Received: 3 May 2018 Accepted: 6 June 2018

Published online: 20 June 2018

\section{References}

1. Döhner H, et al. Acute myeloid. Leukemia. 2015. https://doi.org/10.1056/ NEJMra1406184.

2. Ivaska J, et al. Novel functions of vimentin in cell adhesion, migration, and signaling. Exp Cell Res. 2007:313(10):2050-62.

3. Nieminen $M$, et al. Vimentin function in lymphocyte adhesion and transcellular migration. Nat Cell Biol. 2006;8(2):156-62.

4. Herrmann H, Fouquet B, Franke WW. Expression of intermediate filament proteins during development of Xenopus laevis. I. cDNA clones encoding different forms of vimentin. Development. 1989;105(2):279-98.

5. Schaffeld $M$, et al. Vimentin and desmin of a cartilaginous fish, the shark Scyliorhinus stellaris: sequence, expression patterns and in vitro assembly. Eur J Cell Biol. 2001;80(11):692-702.

6. Belichenko I, Morishima N, Separovic D. Caspase-resistant vimentin suppresses apoptosis after photodynamic treatment with a silicon phthalocyanine in Jurkat cells. Arch Biochem Biophys. 2001;390(1):57-63.

7. Byun $Y$, et al. Caspase cleavage of vimentin disrupts intermediate filaments and promotes apoptosis. Cell Death Differ. 2001;8(5):443-50.

8. Zhang MH, et al. HSP90 protects apoptotic cleavage of vimentin in geldanamycin-induced apoptosis. Mol Cell Biochem. 2006:281(1-2):111-21.

9. Zhu Q-S, Rosenblatt K, Huang K-L, Lahat G, Brobey R, Bolshakov S, Nguyen T, Ding Z, Belousov R, Bill K, Luo X, Lazar A, Dicker A, Mills GB,
Hung M-C, Lev D. Vimentin is a novel AKT1 target mediating motility and invasion. Oncogene. 2011;30(4):457-70

10. Kidd ME, Shumaker DK, Ridge KM. The role of vimentin intermediate filaments in the progression of lung cancer. Am J Respir Cell Mol Biol. 2014;50(1):1-6.

11. Satelli A, Li S. Vimentin in cancer and its potential as a molecular target fo cancer therapy. Cell Mol Life Sci. 2011;68(18):3033-46.

12. Sun B, et al. Role of cellular cytoskeleton in epithelial-mesenchymal transition process during cancer progression. Biomed Rep. 2015:3(5):603-10.

13. Network, T.C.G.A.R. Genomic and epigenomic landscapes of adult de novo acute myeloid leukemia. N Engl J Med. 2013. https://doi. org/10.1056/NEJMoa1301689.

14. Cerami E, Gao J, Dogrusoz U, Gross BE, Sumer SO, Aksoy BA, Jacobsen A Byrne CJ, Heuer ML, Larsson E, Antipin Y, Reva B, Goldberg AP, Sander C, Schultz N. The cBio cancer genomics portal: an open platform for exploring multidimensional cancer genomics data: figure 1. Cancer Discov. 2012;2(5):401-4

15. Gao J, Aksoy BA, Dogrusoz U, Dresdner G, Gross B, Sumer SO, Sun Y, Jacobsen A, Sinha R, Larsson E, Cerami E, Sander C, Schultz N. Integrative analysis of complex cancer genomics and clinical profiles using the cBioPortal. Sci Signal. 2013;6(269):pl1-pl1

16. Metzeler $\mathrm{KH}$, et al. An 86-probe-set gene-expression signature predicts survival in cytogenetically normal acute myeloid leukemia. Blood. 2008;112(10):4193-201.

17. Bullinger $L$, et al. Use of gene-expression profiling to identify prognostic subclasses in adult acute myeloid leukemia. N Engl J Med. 2004:350(16):1605-16.

18. Brabletz T, et al. EMT in cancer. Nat Rev Cancer. 2018;18(2):128.

19. Kahlert UD, Joseph JV, Kruyt FAE. EMT- and MET-related processes in nonepithelial tumors: importance for disease progression, prognosis, and therapeutic opportunities. Mol Oncol. 2017;11(7):860-77.

20. Stavropoulou $V$, et al. MLL-AF9 expression in hematopoietic stem cells drives a highly invasive AML expressing EMT-related genes linked to poor outcome. Cancer Cell. 2016;30(1):43-58.

21. Zou H, et al. Highly methylated genes in colorectal neoplasia: implications for screening. Cancer Epidemiol Biomarkers Prev. 2007;16(12):2686-96

22. Gilles $C$, et al. Transactivation of vimentin by beta-catenin in human breast cancer cells. Cancer Res. 2003;63(10):2658-64.

23. Ysebaert $L$, et al. Expression of beta-catenin by acute myeloid leukemia cells predicts enhanced clonogenic capacities and poor prognosis. Leukemia. 2006:20(7):1211-6.

24. Yeung J, et al. beta-Catenin mediates the establishment and drug resistance of MLL leukemic stem cells. Cancer Cell. 2010;18(6):606-18.
Ready to submit your research? Choose BMC and benefit from:

- fast, convenient online submission

- thorough peer review by experienced researchers in your field

- rapid publication on acceptance

- support for research data, including large and complex data types

- gold Open Access which fosters wider collaboration and increased citations

- maximum visibility for your research: over 100M website views per year

At BMC, research is always in progress.

Learn more biomedcentral.com/submissions 